\title{
Acetylcholine Release in the Mesocorticolimbic Dopamine System during Cocaine Seeking: Conditioned and Unconditioned Contributions to Reward and Motivation
}

\author{
Zhi-Bing You, Bin Wang, Dawnya Zitzman, and Roy A. Wise \\ Behavioral Neuroscience Branch, Intramural Research Program, National Institute on Drug Abuse, National Institutes of Health, Department of Health and \\ Human Services, Baltimore, Maryland 21224
}

\begin{abstract}
Microdialysis was used to assess the contribution to cocaine seeking of cholinergic input to the mesocorticolimbic dopamine system in ventral tegmental area (VTA). VTA acetylcholine (ACh) was elevated in animals lever pressing for intravenous cocaine and in cocaineexperienced and cocaine-naive animals passively receiving similar "yoked" injections. In cocaine-trained animals, the elevations comprised an initial (first hour) peak to $\sim 160 \%$ of baseline and a subsequent plateau of $140 \%$ of baseline for the rest of the cocaine intake period. In cocaine-naive animals, yoked cocaine injections raised ACh levels to the $140 \%$ plateau but did not cause the initial $160 \%$ peak. In cocaine-trained animals that received unexpected saline (extinction conditions) rather than the expected cocaine, the initial peak was seen but the subsequent plateau was absent. VTA ACh levels played a causal role and were not just a correlate of cocaine seeking. Blocking muscarinic input to the VTA increased cocaine intake; the increase in intake offset the decrease in cholinergic input, resulting in the same VTA dopamine levels as were seen in the absence of the ACh antagonists. Increased VTA ACh levels (resulting from $10 \mu \mathrm{M}$ VTA neostigmine infusion) increased VTA dopamine levels and reinstated cocaine seeking in cocaine-trained animals that had undergone extinction; these effects were strongly attenuated by local infusion of a muscarinic antagonist and weakly attenuated by a nicotinic antagonist. These findings identify two cholinergic responses to cocaine self-administration, an unconditioned response to cocaine itself and a conditioned response triggered by cocaine-predictive cues, and confirm that these cholinergic responses contribute to the control of cocaine seeking.
\end{abstract}

Key words: acetylcholine receptor; acetylcholine; ACh; cocaine, dopamine; rat; ventral tegmental area

\section{Introduction}

Cocaine is habit forming primarily because it elevates extracellular dopamine levels at postsynaptic receptors (De Wit and Wise, 1977) in the olfactory tubercle (Ikemoto, 2003), the medial shell of the nucleus accumbens (Carlezon et al., 1995; Ikemoto, 2003), and the prefrontal cortex (Goeders and Smith, 1983). Selfadministered cocaine elevates dopamine levels threefold to fivefold (Pettit and Justice, 1989). The initiation of the next response in a binge can be predicted from the fall from the previous injection of dopamine (Wise et al., 1995) or cocaine (Norman and Tsibulsky, 2006) level.

Because cocaine is an uptake inhibitor and not a releaser (Heikkila et al., 1975), its ability to increase extracellular dopamine depends on the impulse activity in dopaminergic neurons (Nomikos et al., 1990; Yan, 2003). By elevating dopamine levels at the somatodendritic autoreceptor, cocaine tends to inhibit dopaminergic impulse flow (Einhorn et al., 1988). Impulse flow is

\footnotetext{
Received Feb. 15, 2008; revised July 29, 2008; accepted July 29, 2008.

This work was supported by the Intramural Research Program of the National Institute on Drug Abuse, National Institutes of Health, Department of Health and Human Services.

Correspondence should be addressed to Zhi-Bing You, Behavioral Neuroscience Branch, Intramural Research Program, National Institute on Drug Abuse, National Institutes of Health, Department of Health and Human Services, 251 Bayview Boulevard, Baltimore, MD 21224. E-mail: zyou@intra.nida.nih.gov. D0I:10.1523/JNEUROSC1.0694-08.2008

Copyright $\odot 2008$ Society for Neuroscience $\quad$ 0270-6474/08/289021-09\$15.00/0
}

maintained during cocaine self-administration (Pettit and Justice, 1989; Wise et al., 1995), partly because cocaine-predictive stimuli trigger dopaminergic cell firing through ventral tegmental area (VTA) glutamate release (You et al., 2007). However, VTA glutamate release occurs only in early cocaine selfadministration binges; glutamate levels return to normal despite continued cocaine self-administration. Thus, early glutamate peaks do not explain the sustained activation of dopaminergic impulse flow during cocaine self-administration (You et al., 2007).

Another excitatory input to the dopamine system is the cholinergic projection that comes from the laterodorsal (LDTg) and pedunculopontine (PPTg) tegmental nuclei. These sources (Oakman et al., 1995) make synaptic contact with dopaminergic cell bodies (Bolam et al., 1991; Omelchenko and Sesack, 2005). VTA dopaminergic neurons express both nicotinic and muscarinic receptors (Clarke and Pert, 1985; Mash and Potter, 1986; Vilaró et al., 1990; Weiner et al., 1990; Klink et al., 2001) and cholinergic activation in the VTA is associated with increases in VTA dopaminergic firing and terminal dopamine release (Grenhoff et al., 1986; Mereu et al., 1987; Nisell et al., 1994a,b; Blaha et al., 1996; Panagis et al., 1996). Cholinergic input to the VTA has been implicated in the rewarding effects of feeding, lateral hypothalamic brain stimulation, opiates, and psychomotor stimulants (Yeomans et al., 1985; Kofman and Yeomans, 1988; Bechara and 
van der Kooy, 1989; Olmstead et al., 1998; Rada et al., 2000; Nakahara et al., 2001).

In the present study, we monitored fluctuations in acetylcholine (ACh) levels in the VTA of rats self-administering intravenous cocaine, undergoing extinction (saline unexpectedly substituted for cocaine) trials, or passively receiving "yoked" cocaine injections controlled by another animal. To determine whether the observed fluctuations in VTA ACh play causal roles in the mechanism of cocaine seeking (are not just passive correlates), we assessed the effects of VTA cholinergic antagonists on cocaine taking and dopamine levels and we assessed the effects of increased VTA ACh levels (by local cholinesterase inhibition) on dopamine levels and on cocaine seeking under extinction conditions. For the purposes of the present study, we operationally define lever pressing established by a history of reinforcement by intravenous cocaine injections as "cocaine-seeking" behavior. We do not distinguish between cocaine seeking and cocaine taking because the animal had no warning other than reinforcement history as to which trials would be reinforced and which would not.

\section{Materials and Methods \\ Subjects}

One hundred six male Long-Evans rats (Charles River), weighing 350 $400 \mathrm{~g}$ at the time of the surgery, were used. They were housed individually under a reversed light/dark cycle (light on at 8:00 P.M.) with ad libitum access to food and water. They were acclimatized to the new environment for at least for $7 \mathrm{~d}$ before surgery. All procedures were consistent with Principles of Laboratory Animal Care published by National Institutes of Health (publication 86-23; 1996).

\section{Surgery}

Intracranial cannulation. Each rat was anesthetized with pentobarbital (30 mg/kg, i.p.) supplemented with chloral hydrate (140 mg/kg, i.p.), and mounted in a stereotaxic frame. The rat's skull was then exposed and leveled (bregma and lambda at the same elevation: incisor bar $\sim 4.5 \mathrm{~mm}$ below the interaural line), and one or two guide cannulas (CMA/Microdialysis) were implanted above the VTA or the adjacent substantia nigra (SN). Stereotaxic coordinates for the VTA burr holes and cannula entry points were as follows: $5.6 \mathrm{~mm}$ posterior to bregma and $2.2 \mathrm{~mm}$ lateral to the midline. Stereotaxic coordinates for the SN burr holes and cannula entry points were as follows: $5.6 \mathrm{~mm}$ posterior to bregma and $4.0 \mathrm{~mm}$ lateral to the midline. In each case, the guide cannulas were lowered at a $12^{\circ}$ angle toward the midline to a depth of $6.8 \mathrm{~mm}$ from the skull surface and cemented to four stainless-steel screws threaded into the skull. Five groups were implanted with unilateral VTA cannulas, five groups with bilateral VTA cannulas, two groups with bilateral SN cannulas, and two groups with paired ipsilateral VTA and SN cannulas.

Intravenous catheterization. In the same surgery, a small incision was next made to the right of the midline of the neck and the external jugular vein was externalized and an intravenous SILASTIC catheter (Dow Corning) was inserted with its tip reaching the right atrium. The catheter was secured to the vein with silk suture and the other end fed subcutaneously around the back of the neck to exit near the back of the skull. The end was slipped over a bent 22 gauge stainless-steel cannula (Plastics One) with a threaded head used to secure a dummy cannula and, during testing, an infusion line. The catheter and the guide cannula were secured to the head pedestal with dental cement and the wound was sutured.

After surgery, each rat was given $0.25 \mathrm{ml}$ of subcutaneous $2.27 \%$ enrolfloxacine once daily for $3 \mathrm{~d}$ as precaution against infection. The animals were allowed to recover for at least $5 \mathrm{~d}$ before the microdialysis or behavioral training started; during this time, the catheters were flushed daily with $0.2 \mathrm{mg}$ of gentamicin in sterile saline and $0.05 \mathrm{ml}$ of heparin (30 $\mathrm{U} / \mathrm{ml}$ in sterile saline).

\section{Self-administration training}

After recovery from surgery, the animals were assigned to 12 groups of eight animals each. Some animals or data were lost because of catheter or
HPLC failures; final sample sizes varied from six to eight and are indicated in Results. Each rat was connected by polyethylene tubing (protected by a steel coil spring) and a one-channel feed-through swivel to a syringe pump (Razel Scientific) controlled by a microprocessor and placed in an operant chamber (MED Associates) illuminated by a $15 \mathrm{~W}$ red house light and having two response levers $9 \mathrm{~cm}$ above the chamber floor; one lever was fixed and one was retractable. Each rat was trained in $4 \mathrm{~h}$ daily sessions to press the retractable ("active") lever on a FR-1 (fixed ratio 1) schedule of reinforcement for intravenous cocaine or cocaine vehicle. Each session began with the insertion of the active lever into the box; this was the signal of cocaine availability. Cocaine was given in $4.5 \mathrm{~s}$ infusions at unit doses of $1 \mathrm{mg} / \mathrm{kg}$, in $0.13 \mathrm{ml}$ of saline; a white cue light above the lever was illuminated for the duration of each infusion. A time-out period of $20 \mathrm{~s}$ followed each drug infusion; lever presses during this period had no scheduled consequences. Lever presses on the fixed ("inactive") lever were recorded but not rewarded. Experimental testing began after a minimum of $10 \mathrm{~d}$ of stable responding.

Two of the groups served as cocaine-naive control groups; these animals earned only saline infusions $(0.13 \mathrm{ml} /$ active lever press $)$ during the training period (saline-trained). Although lever pressing was minimal during this training condition, the animals received the same handling and exposure to equipment as the cocaine-trained animals. One of these groups was given yoked cocaine infusions, whereas the other was allowed to self-administer saline during the dialysis test day.

\section{Extinction testing}

In preparation for a reinstatement test, one group of cocaine-trained rats was subsequently subjected to a series of daily extinction sessions in which responses on the active lever caused saline $(0.13 \mathrm{ml} /$ injection $)$ rather than cocaine injections. All other conditions were the same as those in the training sessions. This was designed to extinguish the response habit, the conditioned reinforcing association of the cue light, and the discriminative stimulus property of lever insertion. These extinction sessions were continued for $14-18 \mathrm{~d}$, until the rat made $<15$ responses per $4 \mathrm{~h}$ session on the active lever. This criterion corresponded to the baseline level of responding seen in cocaine-naive animals before selfadministration training.

\section{In vivo microdialysis}

After the completion of self-administration or extinction training, each rat was tested in one or more sessions in which microdialysis samples were taken and, in some cases, in which drugs were given by reverse dialysis. The day before microdialysis testing, the blocker was removed from the animal's guide cannula, and a microdialysis probe (CMA/12 14/02; CMA/Microdialysis) was inserted, fixed in position, and connected by FEP tubing through a two- or three-channel feed-through fluid swivel (Instech Laboratories) to a microdialysis pump (CMA/100). The rat was placed in the testing chamber with food and water available ad libitum, and the probe was perfused overnight at a flow rate of $0.4 \mu \mathrm{l} / \mathrm{min}$ with artificial CSF (aCSF) (composition in mM: $148 \mathrm{NaCl}, 2.7 \mathrm{KCl}, 1.2$ $\mathrm{CaCl}_{2}, 0.8 \mathrm{MgCl}_{2}, \mathrm{pH} 7.4$ ). The following day, the perfusion syringes were refilled with aCSF and the flow rate was increased to $1.2 \mu \mathrm{l} / \mathrm{min}$. For animals in experiment 1 , in which ACh levels were to be assayed, $0.2 \mu \mathrm{M}$ neostigmine, a cholinesterase inhibitor, was added to the perfusate to inhibit ACh degradation. The effects of this low concentration of neostigmine, not expected to penetrate the brain in significant concentration, were assessed by comparing control groups from experiment 1 , in which it was used, and experiment 2 , in which it was not. In experiments 2 and 5 , in which dopamine levels were assayed, neostigmine was not included in the perfusate. In experiment 5 , a behaviorally relevant concentration of neostigmine $(10 \mu \mathrm{M})$ was added to the perfusate and its effects on cocaine seeking and on DA levels were assessed.

\section{Biochemical analysis of microdialysis samples}

$A C h$. ACh levels were determined by HPLC. The system consisted of an HPLC pump (ESA 582; ESA), a Unijet microbore ACh/Ch column coupled with a postcolumn immobilized-enzyme reactor (Bioanalytical Systems), a LC-4C amperometric detector equipped with a glassy carbonwired electrode coated with horseradish peroxidase (Bioanalytical Systems), and an ESA model 501 data station (ESA). The potential of the 
peroxidase electrode relative to the $\mathrm{Ag} / \mathrm{AgCl}$ reference electrode was set at $100 \mathrm{mV}$ according the manufacturer's suggestion. The elution of ACh was achieved with a mobile phase consisting of $50 \mathrm{mM} \mathrm{NaH}_{2} \mathrm{PO}_{4}, 0.5 \mathrm{~mm}$ $\mathrm{Na}_{2}$ EDTA, and $0.5 \%$ ProClin, $\mathrm{pH} 8.5$ adjusted with $\mathrm{NaOH}$, pumped at a flow rate of $0.13 \mathrm{ml} / \mathrm{min}$. ACh peaks were quantified by comparison with peak heights of standard ACh solutions. The detection limit was $\sim 5$ $\mathrm{fmol} /$ injection.

Dopamine. Dopamine was measured using a HPLC system coupled to an ESA Coulochem II Detector (model 5200) with a dual-electrode microdialysis cell, and an ESA model 501 data station (ESA). Samples were manually injected onto the column $(3 \mu \mathrm{m}$ particle size; $3 \times 150 \mathrm{~mm}$; Analytical MD-150; ESA). The mobile phase for dopamine separation consisted of $75 \mathrm{~mm} \mathrm{NaH} \mathrm{PO}_{4}, 1.5 \mathrm{~mm}$ OSA, $10 \mu \mathrm{M}$ EDTA, and $8 \%$ acetonitrile, pH 3.0 adjusted with $\mathrm{H}_{3} \mathrm{PO}_{4}$. Dopamine was quantified on both reducing $(-250 \mathrm{mV})$ and oxidizing electrodes $(350 \mathrm{mV})$. The limit of detection for dopamine was $\sim 5 \mathrm{fmol} /$ injection.

\section{Experiment 1: VTA ACh levels during cocaine seeking}

Five groups of animals underwent microdialysis for ACh determinations during various aspects of cocaine seeking. After 60 min equilibration at the new flow rate and subsequent collection of five $10 \mathrm{~min}$ baseline samples, the retractable lever was inserted into the operant chambers. One group of cocaine-trained rats was simply allowed to lever press for intravenous cocaine for $4 \mathrm{~h}$; then the active lever was removed and dialysis sampling was continued for another $2 \mathrm{~h}$. Two groups, one cocainetrained and one cocaine-naive (saline-trained), were given cocaine injections that were yoked to the behavior of an animal that self-administered the drug. Thus, yoked animals received the same total dose and the same pattern of intravenous injections as the ("executive") animals in the selfadministration condition; the critical difference was that the yoked animals did not control their own injections and thus could not predict (as could the executive animals) when to expect the next injection. One group of saline-trained animals were allowed to lever press for intravenous saline (saline self-administration). For animals in these four groups, dialysis samples were taken during the $4 \mathrm{~h}$ period that the executive animals earned cocaine injections, and for an additional 12 samples. Responses on the active and inactive levers and the number and timing of cocaine (or saline) infusions were recorded. The final group of cocainetrained rats was tested in extinction conditions. Here, the lever was inserted but lever pressing resulted in saline rather than cocaine injections. For these animals, responding was monitored and dialysis samples were collected for $150 \mathrm{~min}$.

\section{Experiment 2: cocaine intake and VTA dopamine levels under cholinergic receptor blockade}

Four groups of animals with bilateral cannulas were tested to assess the effects of muscarinic and nicotinic receptor blockade on behavior and on VTA dopamine release. After the completion of self-administration training, these animals were connected to the microdialysis systems using three-channel swivels; one channel was used for intravenous cocaine or saline infusion, whereas the other two channels were used for infusion of the antagonists and for collection of dialysate for assay of dopamine levels. On the testing day, baseline samples were collected for $1 \mathrm{~h}$ and then the rats were allowed to self-administer cocaine for $1 \mathrm{~h}$ with only aCSF in the perfusion medium. Then, for the three experimental groups, the muscarinic antagonist atropine $(100 \mu \mathrm{M})$, the nicotinic antagonist mecamylamine $(100 \mu \mathrm{M})$, or a combination of the two was added to the perfusion medium; aCSF was continued in the untreated control group. Cocaine self-administration was allowed to continue for $3 \mathrm{~h}$ under the antagonist condition. Responses on the active and inactive levers and the numbers of cocaine infusions in the two groups were recorded and compared. Dialysis samples from the left VTA were analyzed for dopamine content.

\section{Experiment 3: effects of VTA atropine on VTA and SN dopamine levels under fixed cocaine levels}

VTA and SN dopamine responses to intravenous cocaine were concurrently determined in two groups, one of which was given atropine (100 $\mu \mathrm{M})$ in the perfusion solution as in experiment 2 . These rats were connected to the microdialysis system after completion of cocaine self- administration training as in experiments 1 and 2. After collection of five baseline samples, intravenous cocaine $(2 \mathrm{mg} / \mathrm{kg})$ was delivered and microdialysis samples were taken for another $80 \mathrm{~min}$. Unlike experiment 2, experiment 3 was designed to reveal the effects of VTA atropine on VTA dopamine levels without the confound of different cocaine levels.

\section{Experiment 4: cocaine intake and SN dopamine levels under cholinergic receptor blockade}

Two groups of animals with bilateral SN cannulas were tested to assess the effects of atropine on behavior and on SN dopamine release. These animals were tested as in experiment 2 , except that atropine $(100 \mu \mathrm{M})$ was the only challenge condition.

\section{Experiment 5: reinstatement of cocaine seeking and VTA} dopamine levels induced by VTA perfusion of high concentration of neostigmine

One group of animals that had been trained to self-administer cocaine subsequently undergone extinction was tested to assess the behavioral and neurochemical effects of increasing VTA ACh levels by way of bilateral VTA infusions of a behaviorally active concentration $(10 \mu \mathrm{M})$ of the cholinesterase inhibitor neostigmine. This concentration was 50 times higher than the concentration that was used in experiment 1 . To assess the effects of local elevations of ACh level on cocaine seeking without the confound of cocaine itself, these animals were tested under extinction conditions. That is, the animals were allowed to lever press, but (as in the previous 14-18 d of extinction "training") only saline was earned in these dialysis sessions. Each rat was tested in four conditions with two tests per day over $2 \mathrm{~d}$. The daily tests were separated by $2 \mathrm{~h}$ of perfusion with aCSF and the order of conditions was counterbalanced. The drugs were included in the perfusion medium after the collection of baseline samples and kept in the perfusion medium until the end of testing. The response lever was inserted for each test $40 \mathrm{~min}$ after initiation of the drug (or vehicle) perfusion. Responses on the previously active and inactive levers were recorded and dialysis samples were collected for dopamine determinations.

\section{Drugs}

Cocaine hydrochloride and the anesthetic used in the surgery were obtained from the pharmacy within the Institute. Neostigmine bromide, atropine, and mecamylamine hydrochloride were purchased from Sigma-Aldrich and dissolved in aCSF. Each drug perfusion solution was adjusted to $\mathrm{pH} 7.4$ before use.

\section{Histology}

After the completion of the microdialysis experiment, the rats were decapitated under deep anesthesia and the brains were removed and fixed in $10 \%$ formalin. Fifty micrometer coronal sections were sliced with a cryostat. The probe location was examined under microscope at low magnification in wet tissue that allowed differentiation of fiber tracts and cell groups. Probe locations were reconstructed on drawings from the atlas of Paxinos and Watson (1998).

\section{Statistical analysis}

VTA ACh and dopamine levels are expressed as the concentrations found in the perfusate (means \pm SEM). Basal values refer to those obtained before drug was added into the perfusion medium or before behavioral testing was initiated. When data were expressed as percentage of baseline values, the mean concentration of the three samples preceding the drug perfusion or the behavioral testing was defined as $100 \%$. The drug effects on behavioral responding were analyzed with two-way ANOVA followed by Fisher's PLSD. The drug effects on ACh and dopamine were analyzed with one-way (within a group) or two-way (between groups) ANOVA with repeated measures over time; Fisher's PLSD test was used for planned between-group comparisons that separated the temporal phases of various treatment groups. Tukey's honestly significant difference (HSD) test was also used when an overall ANOVA did not reveal a significant effect. The three primary phases of the first two experiments were the loading phase, the maintenance phase, and the recovery phase. The loading phase is so named because blood levels of drug are in transition from zero and are thus not yet under pharmacological control (Tsibulsky 


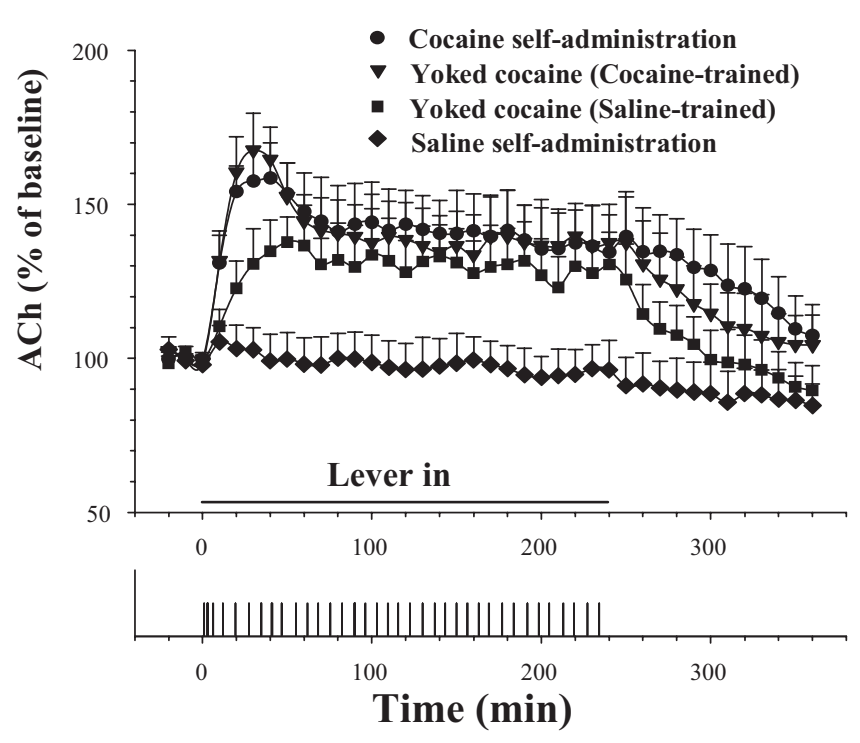

Figure 1. Fluctuations in mean levels of VTA ACh during cocaine self-administration and during yoked cocaine infusions. Significant increases in ACh levels were seen in all three groups receiving cocaine infusions. The increases in ACh during the initial 40 min of drug sessions were significantly higher in cocaine than in saline self-administration-trained groups. Lever pressing for saline had no significant effect on VTA ACh levels in saline self-administration-trained rats. Error bars indicate SEM. A typical response record for cocaine self-administration is shown in the bottom panel.

and Norman, 1999); the first $40 \mathrm{~min}$ of each session were designated the loading phase. The maintenance phase is so named because responding becomes regular and blood levels of the self-administered drug are maintained within a stable range after the initial loading phase (Yokel and Pickens, 1974); the remainder of the self-administration period was designated the maintenance phase. In the first three experiments, dialysis samples were also collected after drug availability was terminated; this was designated the recovery phase.

\section{Results}

Experiment 1: VTA ACh levels during cocaine seeking

Animals earning or receiving yoked cocaine injections had elevated ACh levels for the period of cocaine intake (Fig. 1). Cocaine-trained animals, whether self-administering the drug or receiving yoked injections, showed an increase to a peak of $160 \%$ of baseline during the loading period and a plateau of $140 \%$ of baseline during the maintenance period. Cocaine-naive animals showed an increase during the loading period, but only to the $140 \%$ plateau that was sustained throughout the maintenance period; they did not show the initial $160 \%$ peak seen in the cocaine-experienced animals. Two-way ANOVA showed significant effects of group $\left(F_{(3,23)}=7.48 ; p<0.01\right)$ and time $\left(F_{(38,874)}\right.$ $=22.06 ; p<0.001)$ and group by time interaction $\left(F_{(114,874)}=\right.$ $2.25 ; p<0.001)$. Fishers PSLD test confirmed $(p<0.05)$ that ACh levels were higher for the three groups that received cocaine at each point in the loading period, the maintenance period, and the initial samples in the recovery period, that each point of the $160 \%$ peaks in the two cocaine-trained groups were significantly higher than those of the cocaine-naive group, and that there were no differences between points in the $140 \%$ plateaus among the three cocaine-receiving groups.

In cocaine-trained animals tested under extinction (saline substitution) conditions, ACh levels rose to the same initial $160 \%$ peak elevation as was seen in cocaine-trained animals allowed to self-administer cocaine itself, but was not sustained thereafter at the $140 \%$ plateau (Fig. 2). Two-way ANOVA with repeated mea-

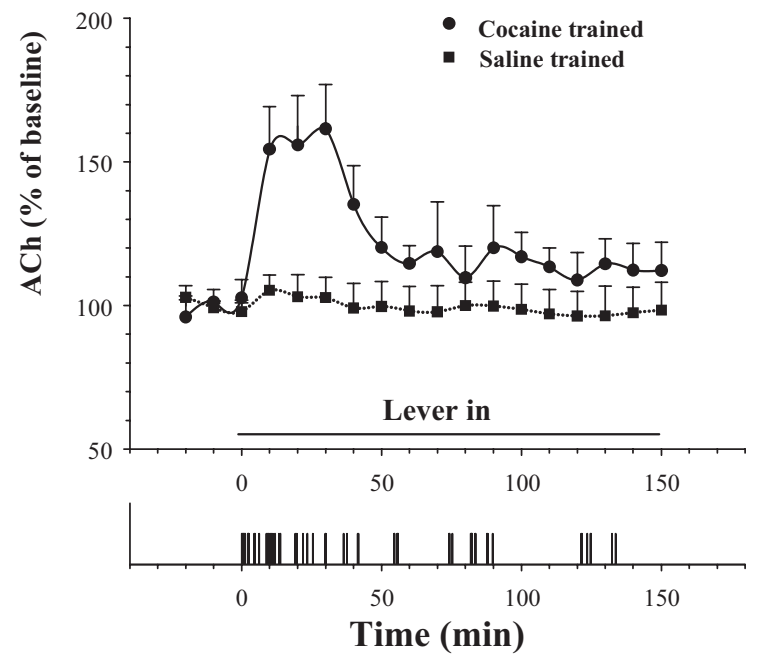

Figure 2. Fluctuations in mean levels of VTA ACh during extinction (saline substituted for cocaine). A typical response record from an extinction session is shown in the bottom panel. Significant increases in VTA ACh were seen during the initial $40 \mathrm{~min}$ of testing in cocaine selfadministration-trained rats. Error bars indicate SEM.

sure over time revealed a significant group by time interaction $\left(F_{(17,221)}=4.14 ; p<0.01\right)$ between cocaine self-administration and saline self-administration trained rats. For cocaine-trained rats, Fisher's PLSD test confirmed $(p<0.05)$ that ACh levels were significantly elevated at all points during the normal loading period but did not remain significantly elevated during the usual maintenance period.

\section{Experiment 2: cocaine intake and VTA dopamine levels under} cholinergic receptor blockade

VTA perfusion of cholinergic antagonists significantly increased the rate of responding on the active lever and, hence, the number of earned cocaine injections (Fig. $3 B$ ). Two-way ANOVA revealed a significant treatment by response interaction $\left(F_{(6,63)}=\right.$ $114.60 ; p<0.001)$. Fisher's PLSD test confirmed $(p<0.05)$ that active lever pressing and number of earned cocaine infusions, but not inactive lever pressing was significantly higher in groups given atropine and atropine plus mecamylamine than in groups given aCSF or mecamylamine alone. No significant differences in lever pressing were found between aCSF and mecamylamine groups.

VTA dopamine levels were elevated to $300 \%$ of baseline during cocaine self-administration under conditions of ACh blockade. Two-way ANOVA with repeated measures comparing groups over time revealed a significant effect of time $\left(F_{(34,714)}=\right.$ 28.46; $p<0.001$ ) but no significant effect of group. Although there was no overall group difference in dopamine levels during the period of cocaine self-administration, note that the effect of atropine and mecamylamine were confounded with betweengroup differences in cocaine intake and level. Fisher's PLSD test and Tukey's HSD test indicate that, once cocaine intake was terminated, dopamine levels dropped more rapidly in the atropine and atropine plus mecamylamine groups.

\section{Experiment 3: effects of VTA atropine on VTA and SN dopamine levels under fixed cocaine levels}

Intravenous administration of cocaine $(2 \mathrm{mg} / \mathrm{kg})$ in cocainetrained rats significantly increased dopamine levels in both the VTA $\left(F_{(10,100)}=15.29 ; p<0.001\right)$ (Fig. 4 , top) and the SN $\left(F_{(10,100)}=11.80 ; p<0.001\right)$ (Fig. 4 , bottom). VTA perfusion of 

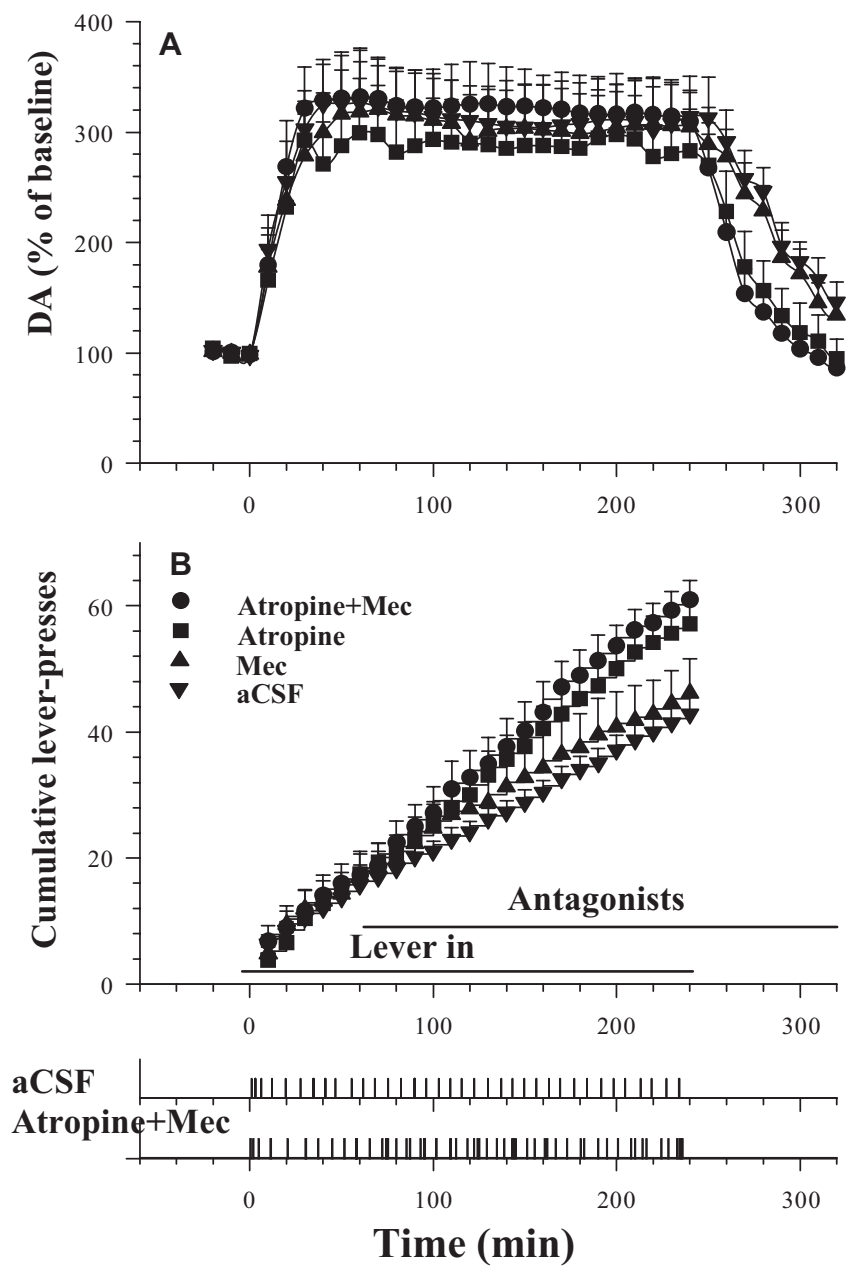

Figure 3. Fluctuations in VTA dopamine levels $(\boldsymbol{A})$ and mean cumulative responses $(\boldsymbol{B})$ as functions of bilateral VTA perfusion of ACh antagonists during cocaine self-administration. VTA perfusion of atropine $(100 \mu \mathrm{M})$ or atropine plus mecamylamine (Atropine $+\mathrm{Mec})$, but not mecamylamine (Mec) $(100 \mu \mathrm{M})$ significantly increased responding for cocaine. Error bars indicate SEM. Event records in the bottom panels show the patterns of active lever responding for an animal receiving aCSF perfusion and an animal receiving Atropine + Mec perfusion. The response lever was inserted at time of 0 ; antagonist perfusions began at time of $60 \mathrm{~min}$.

atropine $(100 \mu \mathrm{M})$ significantly attenuated the cocaine-induced dopamine increase in the VTA (time by treatment interaction; $F_{(10,100)}=2.36 ; p<0.05$ ) but not in the SN (time by treatment interaction; $\left.F_{(10,100)}=0.22 ; p=0.99\right)$.

$\mathrm{SN}$ dopamine levels were significantly elevated during cocaine self-administration in both groups (Fig. 5A). Two-way ANOVA with repeated measures over time revealed a significant effect of time $\left(F_{(34,408)}=24.84 ; p<0.001\right)$ but no significant effect of group $\left(F_{(1,12)}=2.14 ; p=0.17\right)$ and group $\times$ time interaction $\left(F_{(34,408)}=0.63 ; p=0.95\right)$.

Experiment 4: cocaine intake and SN dopamine levels under cholinergic receptor blockade

SN perfusion of atropine (like VTA atropine) significantly increased the rate of responding and the number of cocaine injections (Fig. 5B). Two-way ANOVA revealed a significant treatment by response interaction $\left(F_{(6,63)}=114.60 ; p<0.001\right)$. Fisher's PLSD test confirmed $(p<0.05)$ that active lever pressing and number of earned cocaine infusions, but not inactive lever pressing was significantly higher in animals given atropine than in animals given aCSF.
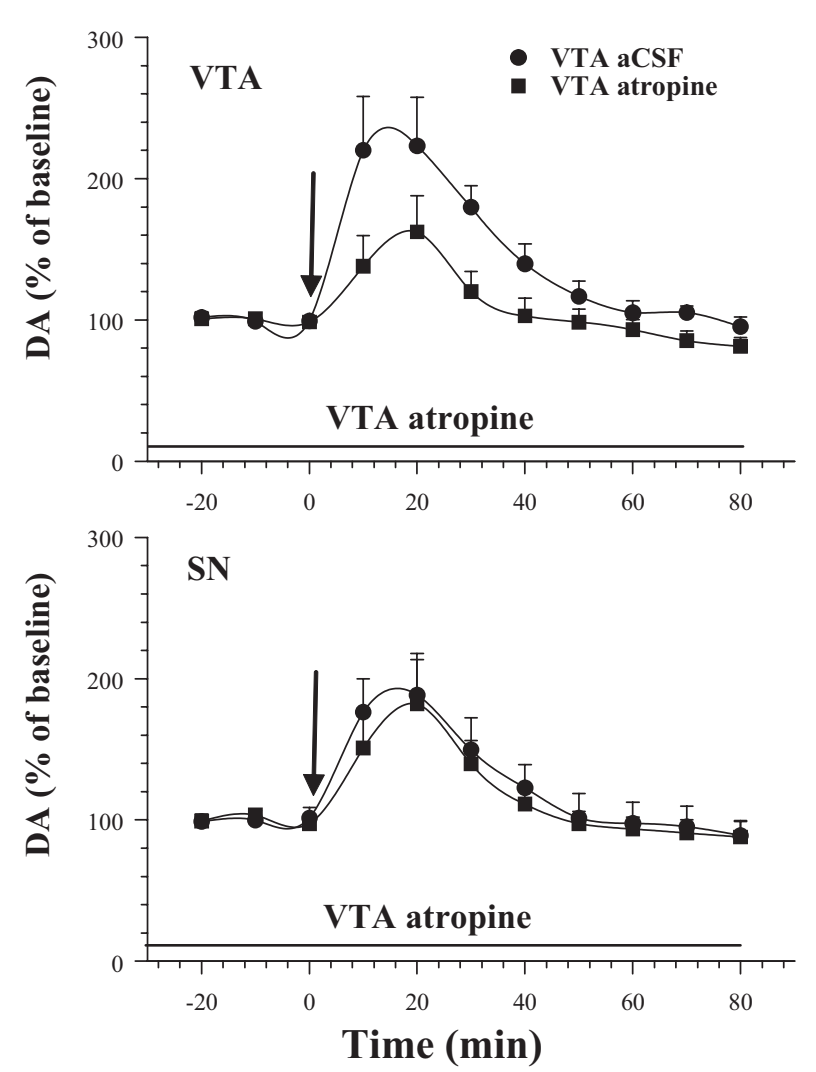

Figure 4. Effects of atropine perfusion into the VTA on cocaine-induced ( $2 \mathrm{mg} / \mathrm{kg}$, i.v.) elevations in VTA and SN dopamine levels. Atropine was perfused through the VTA microdialysis probe before and after the intravenous cocaine injection at time of 0 (arrow). VTA atropine perfusion significantly attenuated the local (VTA) but not the distal (SN) effect of intravenous cocaine. Error bars indicate SEM.

Experiment 5: cocaine seeking and VTA dopamine levels induced by VTA perfusion of high concentration of neostigmine after extinction trials

Elevation of ACh levels by perfusion of $10 \mu \mathrm{M}$ neostigmine reinstated nonrewarded responding in cocaine-trained animals after 2 weeks of testing in extinction conditions; this effect was attenuated in animals given VTA infusions of cholinergic antagonists (Fig. 6A). Two-way ANOVA revealed a significant treatment by lever interaction $\left(F_{(6,63)}=114.6 ; p<0.001\right)$. Fisher's PLSD tests confirmed $(p<0.05)$ that active lever responding was significantly higher in animals treated with VTA infusion of neostigmine and neostigmine plus mecamylamine than in control animals perfused only with aCSF or in animals treated with the combination of neostigmine plus atropine and that responding was significantly lower in the neostigmine plus mecamylamine group than in the neostigmine alone group. There were no significant between-group differences in responding on the inactive lever.

Comparison of the response rates between experiments 1 and 3 revealed no significant effect of the VTA perfusion of the $0.2 \mu \mathrm{M}$ concentration of neostigmine that was used in the early $\mathrm{ACh}$ determinations. Mean number of active-lever responses for the cocaine self-administration group in experiment 1 (which was perfused with $0.2 \mu \mathrm{M}$ neostigmine, for ACh determination) and mean responses on the active lever for the cocaine selfadministration group in experiment 2 (which was perfused only with aCSF, for dopamine determination) were $40.6 \pm 3.1$ and $42.8 \pm 2.6$, respectively $\left(F_{(1,12)}=2.67 ; \mathrm{NS}\right)$. 

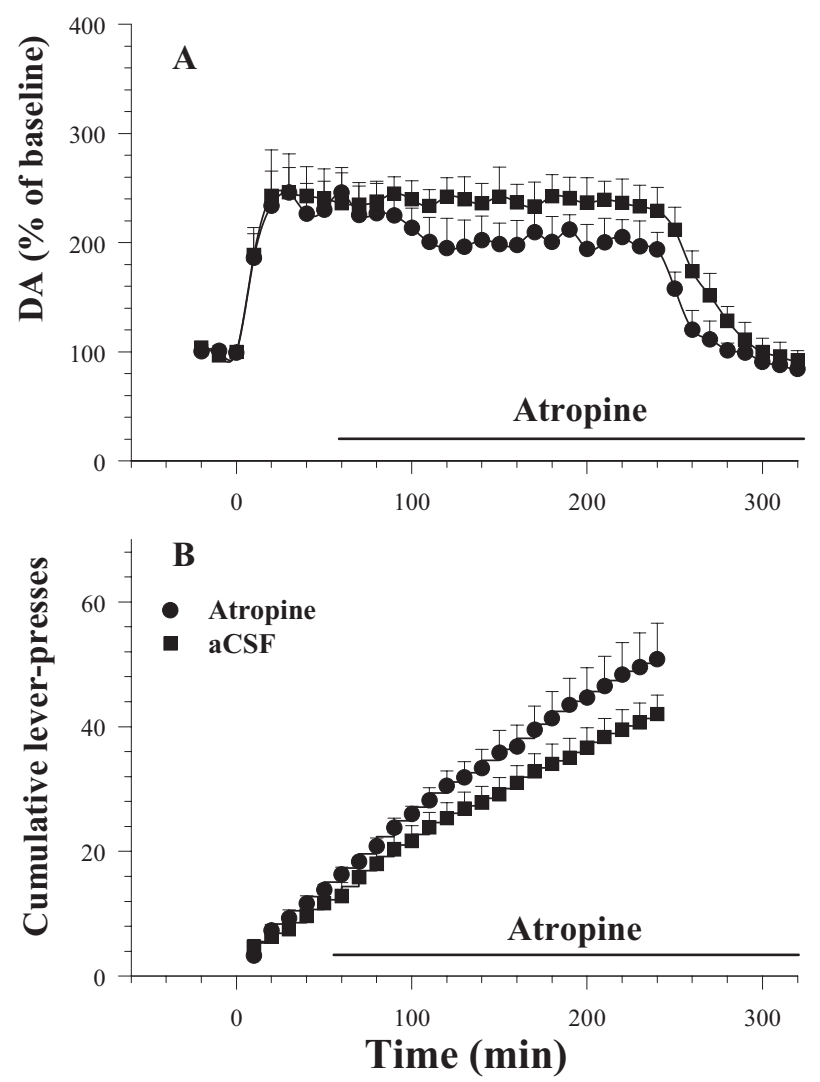

Figure 5. Fluctuations in SN dopamine levels $(\boldsymbol{A})$ and mean cumulative responses $(\boldsymbol{B})$ as functions of bilateral SN perfusion of atropine during cocaine self-administration. SN perfusion of atropine (100 $\mu \mathrm{M})$ significantly increased responding for cocaine. The response lever was inserted at time of 0 ; atropine perfusion began at time of $60 \mathrm{~min}$. Error bars indicate SEM.

VTA perfusion of the higher, $10 \mu \mathrm{M}$, concentration of neostigmine elevated dopamine levels and this effect was antagonized by each of the two ACh antagonists (Fig. 5B). Two-way ANOVA revealed a significant time by treatment interaction $\left(F_{(48,320)}=\right.$ $2.91 ; p<0.001)$. Fisher's PLSD tests confirmed that dopamine levels of animals given VTA perfusion of $10 \mu \mathrm{M}$ neostigmine alone were significantly higher than those of animals given VTA perfusion of aCSF alone or of $10 \mu \mathrm{M}$ neostigmine plus atropine; that those given $10 \mu \mathrm{M}$ neostigmine plus atropine were not significantly different from those given VTA perfusion of aCSF alone; and that those of animals given VTA perfusion of $10 \mu \mathrm{M}$ neostigmine plus mecamylamine were significantly lower than those of animals given VTA perfusion of $10 \mu \mathrm{M}$ neostigmine alone but significantly higher than those given aCSF alone or 10 $\mu \mathrm{M}$ neostigmine plus atropine.

\section{Histological examination of the microdialysis membrane locations}

Probe placements are shown in Figure 7. The probe membranes of rats with unilateral or bilateral VTA probes were primarily in the posterior VTA, usually penetrating the parabrachial pigmented and paranigral nuclei, and occasionally partially intruding the rostral interpeduncular nucleus. The SN probe membranes penetrated both the zona compacta and the zona reticulata of SN. One rat in the experienced yoked group was excluded because of dorsal probe placements; ACh levels were lower and not reliably measured. Two rats used in experiment 2 (one in mecamylamine perfusion group one in atropine perfusion group) were excluded because of dorsal placement of one
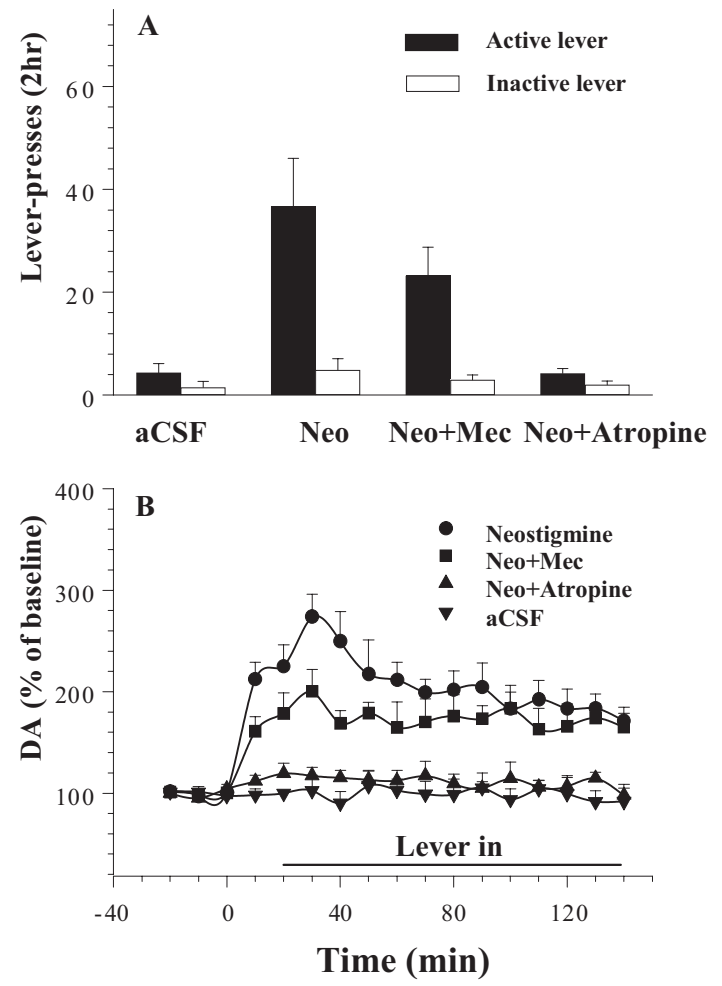

Figure 6. Effects of bilateral VTA perfusion of neostigmine (Ne0; $10 \mu \mathrm{m})$ on lever pressing $(\boldsymbol{A})$ and VTA dopamine levels (B) under nicotinic [mecamylamine (Mec), $100 \mu \mathrm{M}$ ], and muscarinic (atropine, $100 \mu \mathrm{m}$ ) blockade after extinction trials in cocaine-trained animals. Neostigmine perfusion significantly increased responding and VTA dopamine levels; these effects were each significantly attenuated by mecamylamine and blocked by atropine. Error bars indicate SEM.

probe in each rat. One rat in experiment 3 was excluded because the microdialysis line broke during testing.

\section{Discussion}

The present study reveals two sources of fluctuation of cholinergic input to the VTA during cocaine seeking and cocaine taking and confirms that these alterations in cholinergic input influence (are part of the mechanism underlying) cocaine-seeking behavior.

Two sources of VTA ACh fluctuation during cocaine seeking Two cholinergic sources were identified. The first source (associated with cocaine seeking but not dependent on the actual delivery of cocaine) is responsible for the $160 \%$ initial peak in ACh that is triggered by insertion of the response lever into the operant chamber of a cocaine-trained but not a cocaine-naive animal. This is a conditioned response, a response that depends on the animal's recent reinforcement history in the presence of the response lever and other cocaine-associated contextual cues. This peak is seen when the animal can reasonably be assumed to expect cocaine, even if cocaine is not actually earned on the trial in question [for example, the first extinction trial, in which cocaine has always been given in the past (last training trials) but unexpected saline is now substituted for the expected cocaine].

The second source of elevated ACh is cocaine itself, which is responsible for the bulk of the $140 \%$ elevation that is sustained throughout the session. This response is unconditioned, because it is seen equally in cocaine-trained and cocaine-naive animals whether they earn cocaine or receive it passively. This sustained 


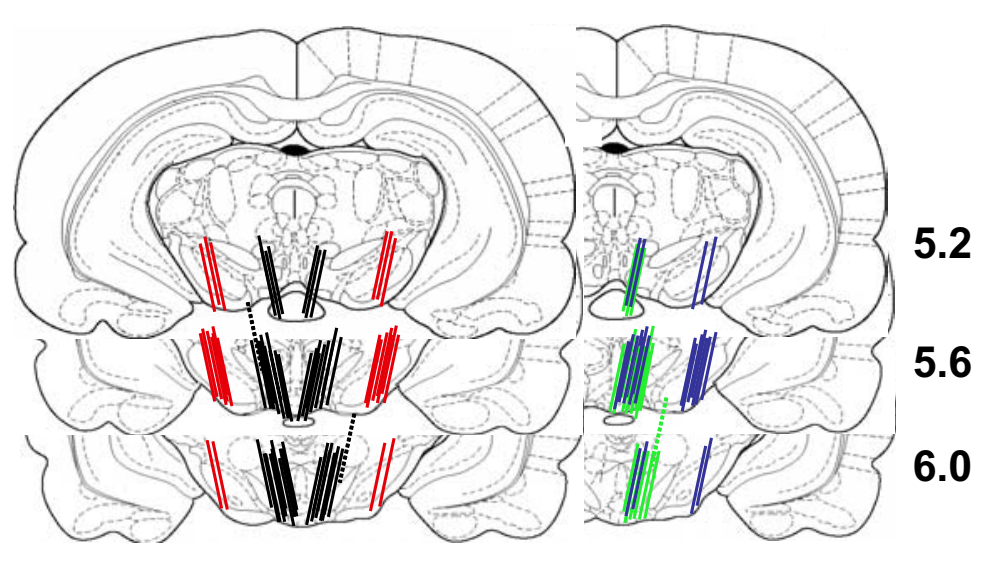

Figure 7. Probe placements. The lines indicate the location of the active portion of the dialysis membrane. Bilateral placements for VTA (black) and SN (red) placements are shown in the left panels; unilateral VTA placements (green) and dual VTA-SN probe placements (blue) are shown in the right panels. The dotted lines indicate the dorsal placements in rats that were excluded from statistical analysis. The number of lines is less than the number of animals because of overlap in the placements. The drawings were adapted from the atlas of Paxinos and Watson (1998). The numbers on the right side of the drawings indicate the distance (in millimeters) posterior to the bregma.

elevation in VTA ACh levels contributes to the rewarding effect of the drug by increasing dopaminergic impulse flow as reflected in increased VTA dopamine levels. The increased VTA dopamine levels reflect dendritic dopamine release (Geffen et al., 1976) [associated with dopaminergic impulse flow (Legault and Wise, 1999; Legault et al., 2000)] and contribute to the rewarding effects of the cocaine (Ranaldi and Wise 2001). The sustained VTA ACh release appears to reflect a transsynaptic positive feedback from other cocaine-sensitive brain regions. This feedback signal opposes the negative feedback mediated by the local activation of autoreceptors.

The source of the ACh appears to be the pons. Cholinergic input to the VTA comes from two brainstem nuclei: LDTg and the caudomedial portion of PPTg (Woolf and Butcher, 1986; Oakman et al., 1995). Cholinergic neurons comprise a minority of the neurons in LDTg and PPTg (Omelchenko and Sesack, 2005), which also contains glutamatergic (Geisler et al., 2007) and GABAergic (Ford et al., 1995) neurons. The cholinergic components of the nuclei are traditionally identified with the ascending reticular activating system (Steriade et al., 1990; Jones, 2005). They project to the thalamus and a range of forebrain structures (Woolf and Butcher, 1986; Gould et al., 1989; Charara et al., 1996), but are not part of the basal forebrain cholinergic system that innervates the cortex (Woolf et al., 1986; Wainer and Mesulam, 1990). Cholinergic inputs target both dopaminergic and GABAergic neurons in the VTA, making asymmetric, presumed excitatory, synapses on dopaminergic neurons projecting to nucleus accumbens and prefrontal cortex and on GABAergic neurons projecting to prefrontal cortex and also making symmetric, presumably inhibitory, synapses on both dopaminergic and GABAergic projections to nucleus accumbens and prefrontal cortex (Omelchenko and Sesack, 2006). PPTg cells respond to sensory stimuli, and do so with much shorter latency than do VTA dopaminergic neurons (Pan and Hyland, 2005). Although PPTg neurons do not respond exclusively to reward-predictive stimuli, input from PPTg is essential to the ability of putative dopaminergic VTA neurons to do so (Pan and Hyland, 2005).

Atropine infused through the VTA probe decreased VTA but not SN DA levels, although each was elevated by cocaine. This suggests that VTA infusion of atropine acted locally and did not diffuse sufficiently to act by blocking cholinergic input to the SN.
This was a weak test of diffusion, however, because the two probe shafts were $1.2 \mathrm{~mm}$ apart and could not be put closer to one another without causing significant local damage. The more interesting finding from animals with $\mathrm{SN}$ probes was that selfadministered cocaine caused elevation of $\mathrm{SN}$ as well as VTA dopamine levels, and atropine infused into the $\mathrm{SN}$, like atropine infused into the VTA, increased cocaine self-administration. A detailed analysis of the cholinergic input to the $\mathrm{SN}$ during cocaine self-administration was beyond the scope of the present study but will be taken up in some detail in future studies. A potential role for $\mathrm{ACh}$ in $\mathrm{SN}$ is of particular interest to us because of two relatively unnoticed findings suggesting VTA is not uniquely involved in reward function. First, it was reported decades ago that electrical stimulation of pars compacta and/or pars lateralis of the substantia nigra is rewarding (Routtenberg and Malsbury, 1969; Corbett and Wise, 1980; Wise, 1981). Second, blockade of $\mathrm{D}_{1}$ dopamine receptors in SN increases cocaine intake (Quinlan et al., 2004), as does blockade of $\mathrm{D}_{1}$ dopamine receptors in the VTA (Ranaldi and Wise, 2001).

Muscarinic receptors appear to play the major role. The present data fit well with other reports (Yeomans and Baptista, 1997; Ikemoto, 2003) suggesting that, although nicotinic and muscarinic input each contribute to dopaminergic activation, muscarinic activation makes the stronger contribution. Midbrain dopamine neurons express both nicotinic (Wada et al., 1989, 1990) and muscarinic (Vilaró et al., 1990; Weiner et al., 1990) receptors and electrical stimulation of cholinergic input to the VTA activates the dopamine system with contributions through each (Lester et al., 2008). The nicotinic contribution is limited, however, by rapid desensitization of the receptor (Pidoplichko et al., 1997). Thus, whereas nicotinic and muscarinic activation each contribute to the early peak in the present study, the sustained elevations are likely to be primarily muscarinic. This suggestion fits well with the finding that stimulation of the cholinergic input to the VTA causes a short-lasting activation that is nicotinic and a long tonic activation that is muscarinic in origin (Forster and Blaha, 2000; Forster et al., 2002). Against this interpretation, however, is our finding that nicotinic blockade caused a small compensatory increase in cocaine self-administration that was consistent throughout the session and not limited to the early conditioned dopamine elevation. Because the rate of responding in this paradigm is dependent on dopamine levels just before each lever press, $\sim 5$ min after the previous cocaine injection, there was apparently at least some degree of tonic nicotinic contribution to dopaminergic activation. Nonetheless, the primary cholinergic input to the reward system in this and other studies seems to be muscarinic.

\section{ACh fluctuations play a causal role in cocaine seeking}

ACh fluctuations do not merely correlate with aspects of cocaine seeking; they play a role in the mechanism of the behavior. Cholinergic blockade increased responding that was reinforced by earned cocaine. Similar increases are seen when the dose of cocaine per injection is reduced (Pickens and Thompson, 1968), when the dopamine system is blocked (De Wit and Wise, 1977), 
or when glutamatergic input to the VTA is blocked (You et al., 2007). The tentative interpretation of such increases is that they reflect reduced rewarding efficacy (or, more precisely, reduced satiating efficacy) of the drug. However, cholinergic blockade decreased responding under extinction conditions. In the case of responding under extinction conditions, blocking the excitatory glutamatergic input to the VTA has similar effects to blocking the excitatory cholinergic input: although increasing responding when cocaine is available, it decreases responding dramatically in extinction (You et al., 2007). Here, our working hypothesis is that the treatment blocks the effectiveness of the conditioned reinforcers and discriminative stimuli that sustain responding in extinction. In the present case, this could be the presence of the response lever that, during training, had signaled cocaine availability, or it could be the cue light that continued to be illuminated when the animals lever pressed in extinction. The mechanism would be straightforward: Decreasing the excitatory input to the dopamine system reduces the extracellular dopamine impinging on the cocaine-blocked uptake mechanism.

The initial $160 \%$ peak in ACh was always accompanied by lever pressing, but it seems unlikely that it was a consequence of simple motor output. The sources of cholinergic input to the VTA are responsive with short latency to sensory stimuli and respond to reward-predictive stimuli more rapidly than do VTA dopamine neurons (Pan and Hyland, 2005). Thus, the initial $160 \%$ ACh peak was most likely a consequence of the sensory input associated with insertion of the response lever into the test chamber, the illumination of the injection-associated cue light, or both. Separating the consequences of the two stimulus sources will be an important but challenging task because the dopaminergic response to reward-associated sensory cues is complex. Unearned presentation of cocaine-predictive cues has no effect on dopamine levels in the dorsal striatum (Ito et al., 2002) or the shell of nucleus accumbens (Ito et al., 2000) but elevates dopamine levels in the core of nucleus accumbens (Ito et al., 2000). Earned (response contingent) presentation of cocaine-predictive cues elevate dopamine levels in the dorsal striatum (Ito et al., 2002) but not the core or shell of nucleus accumbens (Ito et al., 2000). The kind of cue (unearned discriminative stimulus or earned conditioned reinforcers) may determine what subset of cholinergic neurons respond and what subregion of VTA receives ACh under various conditions. Moreover, a more rapid detection system than microdialysis will be needed to tease apart the effects of various cue conditions on VTA ACh release.

VTA ACh release appears to be significant both for the motivation to obtain cocaine and for the rewarding effect of the earned drug. VTA ACh is implicated in reward function because cholinergic receptor blockade in the VTA caused compensatory increases in self-administered cocaine intake. When cholinergic receptor blockers were given, the animals adjusted their cocaine intake upward by an amount just sufficient to maintain the same VTA dopamine levels as were earned in the absence of cholinergic receptor blockade. Cocaine intake under these conditions is known to be regulated; the animal makes compensatory increases in cocaine intake when the dose per injection is decreased and compensatory increases in lever pressing when the response requirement is increased (Pickens and Thompson, 1968; Gerber and Wise, 1989). Although closely regulated in normal animals (Tsibulsky and Norman, 1999), cocaine intake is increased when the rewarding effect of cocaine is compromised by blockade of dopamine neurotransmission (De Wit and Wise, 1977). The increased intake under cholinergic blockade in the present experiment, and the fact that the increased intake was just sufficient to maintain the same elevation in VTA dopamine concentration as was seen in untreated animals, suggests that VTA ACh contributes to the rewarding effects of cocaine. Several lines of evidence similarly implicate VTA ACh in the rewarding effects of lateral hypothalamic brain stimulation (Yeomans et al., 1985; Kofman and Yeomans, 1988). Cholinergic and glutamatergic (You et al., 2007) afferents to the VTA thus appear to be important segments of brain reward circuitry.

\section{References}

Bechara A, van der Kooy D (1989) The tegmental pedunculopontine nucleus: a brain-stem output of the limbic system critical for the conditioned place preferences produced by morphine and amphetamine. J Neurosci 9:3400-3409.

Blaha CD, Allen LF, Das S, Inglis WL, Latimer MP, Vincent SR, Winn P (1996) Modulation of dopamine efflux in the nucleus accumbens after cholinergic stimulation of the ventral tegmental area in intact, pedunculopontine tegmental nucleus-lesioned, and laterodorsal tegmental nucleus-lesioned rats. J Neurosci 16:714-722.

Bolam JP, Francis CM, Henderson Z (1991) Cholinergic input to dopaminergic neurons in the substantia nigra: a double immunocytochemical study. Neuroscience 41:483-494.

Carlezon WA Jr, Devine DP, Wise RA (1995) Habit-forming actions of nomifensine in nucleus accumbens. Psychopharmacology 122:194-197.

Charara A, Smith Y, Parent A (1996) Glutamatergic inputs from the pedunculopontine nucleus to midbrain dopaminergic neurons in primates: Phaseolus vulgaris-leucoagglutinin anterograde labeling combined with postembedding glutamate and GABA immunohistochemistry. J Comp Neurol 364:254-266.

Clarke PB, Pert A (1985) Autoradiographic evidence for nicotine receptors on nigrostriatal and mesolimbic dopaminergic neurons. Brain Res 348:355-358.

Corbett D, Wise RA (1980) Intracranial self-stimulation in relation to the ascending dopaminergic systems of the midbrain: a moveable electrode mapping study. Brain Res 185:1-15.

De Wit H, Wise RA (1977) Blockade of cocaine reinforcement in rats with the dopamine receptor blocker pimozide, but not with the noradrenergic blockers phentolamine or phenoxybenzamine. Can J Psychol 31:195-203.

Einhorn LC, Johansen PA, White FJ (1988) Electrophysiological effects of cocaine in the mesoaccumbens dopamine system: studies in the ventral tegmental area. J Neurosci 8:100-112.

Ford B, Holmes CJ, Mainville L, Jones BE (1995) GABAergic neurons in the rat pontomesencephalic tegmentum: codistribution with cholinergic and other tegmental neurons projecting to the posterior lateral hypothalamus. J Comp Neurol 363:177-196.

Forster GL, Blaha CD (2000) Laterodorsal tegmental stimulation elicits dopamine efflux in the rat nucleus accumbens by activation of acetylcholine and glutamate receptors in the ventral tegmental area. Eur J Neurosci 12:3596-3604.

Forster GL, Yeomans JS, Takeuchi J, Blaha CD (2002) M5 muscarinic receptors are required for prolonged accumbal dopamine release after electrical stimulation of the pons in mice. J Neurosci 22:RC190(1-6).

Geffen LB, Jessell TM, Cuello AC, Iversen LL (1976) Release of dopamine from dendrites in rat substantia nigra. Nature 260:258-260.

Geisler S, Derst C, Veh RW, Zahm DS (2007) Glutamatergic afferents of the ventral tegmental area in the rat. J Neurosci 27:5730-5743.

Gerber GJ, Wise RA (1989) Pharmacological regulation of intravenous cocaine and heroin self-administration in rats: a variable dose paradigm. Pharmacol Biochem Behav 32:527-531.

Goeders NE, Smith JE (1983) Cortical dopaminergic involvement in cocaine reinforcement. Science 221:773-775.

Gould E, Woolf NJ, Butcher LL (1989) Cholinergic projections to the substantia nigra from the pedunculopontine and laterodorsal tegmental nuclei. Neuroscience 28:611-623.

Grenhoff J, Aston-Jones G, Svensson TH (1986) Nicotinic effects on the firing pattern of midbrain dopamine neurons. Acta Physiol Scand 128:351-358.

Heikkila RE, Orlansky H, Cohen G (1975) Studies on the distinction between uptake inhibition and release of $\left({ }^{3} \mathrm{H}\right)$ dopamine in rat brain tissue slices. Biochem Pharmacol 24:847-852. 
Ikemoto S (2003) Involvement of the olfactory tubercle in cocaine reward: intracranial self-administration studies. J Neurosci 23:9305-9311.

Ito R, Dalley JW, Howes SR, Robbins TW, Everitt BJ (2000) Dissociation in conditioned dopamine release in the nucleus accumbens core and shell in response to cocaine cues and during cocaine-seeking behavior in rats. J Neurosci 20:7489-7495.

Ito R, Dalley JW, Robbins TW, Everitt BJ (2002) Dopamine release in the dorsal striatum during cocaine-seeking behavior under the control of drug-associated cue. J Neurosci 22:6247-6253.

Jones BE (2005) Basic mechanisms of sleep-wake states. In: Principles and practice of sleep medicine, Ed 4 (Kryger MH, Roth T, Dement WC, eds), pp 136-153. New York: Saunders.

Klink R, de Kerchove d'Exaerde A, Zoli M, Changeux JP (2001) Molecular and physiological diversity of nicotinic acetylcholine receptors in the midbrain dopaminergic nuclei. J Neurosci 21:1452-1463.

Kofman O, Yeomans JS (1988) Cholinergic antagonists in ventral tegmentum elevate thresholds for lateral hypothalamic and brainstem selfstimulation. Pharmacol Biochem Behav 31:547-559.

Legault M, Wise RA (1999) Injections of N-methyl-D-aspartate into the ventral hippocampus increase extracellular dopamine in the ventral tegmental area and nucleus accumbens. Synapse 31:241-249.

Legault M, Rompré PP, Wise RA (2000) Chemical stimulation of the ventral hippocmpus elevates nucleus accumbens dopamine by activating dopaminergic neurons of the ventral tegmental area. J Neurosci 20:1635-1642.

Lester DB, Miller AD, Pate TD, Blaha CD (2008) Midbrain acetylcholine and glutamate receptors modulate accumbal dopamine release. Neuroreport 19:991-995.

Mash DC, Potter LT (1986) Autoradiographic localization of M1 and M2 muscarine receptors in the rat brain. Neuroscience 19:551-564.

Mereu G, Yoon KW, Boi V, Gessa GL, Naes L, Westfall TC (1987) Preferential stimulation of ventral tegmental area dopaminergic neurons by nicotine. Eur J Pharmacol 141:395-399.

Nakahara D, Ishida Y, Nakamura M, Furuno N, Nishimori T (2001) Intracranial self-stimulation induces Fos expression in GABAergic neurons in the rat mesopontine tegmentum. Neuroscience 106:633-641.

Nisell M, Nomikos GG, Svensson TH (1994a) Systemic nicotine-induced dopamine release in the rat nucleus accumbens is regulated by nicotinic receptors in the ventral tegmental area. Synapse 16:36-44.

Nisell M, Nomikos GG, Svensson TH (1994b) Infusion of nicotine in the ventral tegmental area or the nucleus accumbens of the rat differentially affects accumbal dopamine release. Pharmacol Toxicol 75:348-352.

Nomikos GG, Damsma G, Wenkstern D, Fibiger HC (1990) In vivo characterization of locally applied dopamine uptake inhibitors by striatal microdialysis. Synapse 6:106-112.

Norman AB, Tsibulsky VL (2006) The compulsion zone: a pharmacological theory of acquired cocaine self-administration. Brain Res 1116:143-152.

Oakman SA, Faris PL, Kerr PE, Cozzari C, Hartman BK (1995) Distribution of pontomesencephalic cholinergic neurons projecting to substantia nigra differs significantly from those projecting to ventral tegmental area. J Neurosci 15:5859-5869.

Olmstead MC, Munn EM, Franklin KB, Wise RA (1998) Effects of pedunculopontine tegmental nucleus lesions on responding for intravenous heroin under different schedules of reinforcement. J Neurosci 18:5035-5044.

Omelchenko N, Sesack SR (2005) Laterodorsal tegmental projections to identified cell populations in the rat ventral tegmental area. J Comp Neurol 483:217-235.

Omelchenko N, Sesack SR (2006) Cholinergic axons in the rat ventral tegmental area synapse preferentially onto mesoaccumbens dopamine neurons. J Comp Neurol 494:863-875.

Pan WX, Hyland BI (2005) Pedunculopontine tegmental nucleus controls conditioned responses of midbrain dopamine neurons in behaving rats. J Neurosci 25:4725-4732.

Panagis G, Nisell M, Nomikos GG, Chergui K, Svensson TH (1996) Nicotine injections into the ventral tegmental area increase locomotion and Fos-like immunoreactivity in the nucleus accumbens of the rat. Brain Res 730:133-142.

Paxinos G, Watson C (1998) The rat brain in stereotaxic coordinates, Ed 4. San Diego: Academic.
Pettit HO, Justice JB Jr (1989) Dopamine in the nucleus accumbens during cocaine self-administration as studied by in vivo microdialysis. Pharmacol Biochem Behav 34:899-904.

Pickens R, Thompson T (1968) Cocaine-reinforced behavior in rats: effects of reinforcement magnitude and fixed-ratio size. J Pharmacol Exp Ther 161:122-129.

Pidoplichko VI, DeBiasi M, Williams JT, Dani JA (1997) Nicotine activates and desensitizes midbrain dopamine neurons. Nature 390:401-404.

Quinlan MG, Sharf R, Lee DY, Wise RA, Ranaldi R (2004) Blockade of substantia nigra dopamine D1 receptors reduces intravenous cocaine reward in rats. Psychopharmacology 175:53-59.

Rada PV, Mark GP, Yeomans JJ, Hoebel BG (2000) Acetylcholine release in ventral tegmental area by hypothalamic self-stimulation, eating, and drinking. Pharmacol Biochem Behav 65:375-379.

Ranaldi R, Wise RA (2001) Blockade of $\mathrm{D}_{1}$ dopamine receptors in the ventral tegmental area decreases cocaine reward: possible role for dendritically released dopamine. J Neurosci 21:5841-5846.

Routtenberg A, Malsbury C (1969) Brainstem pathways of reward. J Comp Physiol Psychol 68:22-30.

Steriade M, Datta S, Paré D, Oakson G, Curró Dossi RC (1990) Neuronal activities in brain-stem cholinergic nuclei related to tonic activation processes in thalamocortical systems. J Neurosci 10:2541-2559.

Tsibulsky VL, Norman AB (1999) Satiety threshold: a quantitative model of maintained cocaine self-administration. Brain Res 839:85-93.

Vilaró MT, Palacios JM, Mengod G (1990) Localization of m5 muscarinic receptor mRNA in rat brain examined by in situ hybridization histochemistry. Neurosci Lett 114:154-159.

Wada E, Wada K, Boulter J, Deneris E, Heinemann S, Patrick J, Swanson LW (1989) Distribution of alpha 2, alpha 3, alpha 4, and beta 2 neuronal nicotinic receptor subunit mRNAs in the central nervous system: a hybridization histochemical study in the rat. J Comp Neurol 284:314-335.

Wada E, McKinnon D, Heinemann S, Patrick J, Swanson LW (1990) The distribution of mRNA encoded by a new member of the neuronal nicotinic acetylcholine receptor gene family (alpha 5 ) in the rat central nervous system. Brain Res 526:45-53.

Wainer BH, Mesulam MM (1990) Ascending cholinergic pathways in the rat brain. In: Brain cholinergic systems (Steriade M, Biesold D, eds), pp 65-119. New York: Oxford UP.

Weiner DM, Levey AI, Brann MR (1990) Expression of muscarinic acetylcholine and dopamine receptor mRNAs in rat basal ganglia. Proc Natl Acad Sci U S A 87:7050-7054.

Wise RA (1981) Intracranial self-stimulation: mapping against the lateral boundaries of the dopaminergic cells of the substantia nigra. Brain Res 213:190-194.

Wise RA, Newton P, Leeb K, Burnette B, Pocock D, Justice JB Jr (1995) Fluctuations in nucleus accumbens dopamine concentration during intravenous cocaine self-administration in rats. Psychopharmacology 120:10-20.

Woolf NJ, Butcher LL (1986) Cholinergic systems in the rat brain: III. Projections from the pontomesencephalic tegmentum to the thalamus, tectum, basal ganglia, and basal forebrain. Brain Res Bull 16:603-637.

Woolf NJ, Hernit MC, Butcher LL (1986) Cholinergic and non-cholinergic projections from the rat basal forebrain revealed by combined choline acetyltransferase and Phaseolus vulgaris leucoagglutinin immunohistochemistry. Neurosci Lett 66:281-286.

Yan QS (2003) Involvement of non-exocytotic mechanisms in ethanolinduced in vivo dopamine release: comparisons with cocaine. Eur J Pharmacol 477:37-44.

Yeomans J, Baptista M (1997) Both nicotinic and muscarinic receptors in ventral tegmental area contribute to brain-stimulation reward. Pharmacol Biochem Behav 57:915-921.

Yeomans JS, Kofman O, McFarlane V (1985) Cholinergic involvement in lateral hypothalamic rewarding brain stimulation. Brain Res 329:19-26.

Yokel RA, Piekens R (1974) Drug level of D- and L-amphetamine during intravenous self-administration. Psychopharmacologia 34:255-264.

You ZB, Wang B, Zitzman D, Azari S, Wise RA (2007) A role for conditioned ventral tegmental glutamate release in cocaine-seeking. J Neurosci 27:10546-10555. 